Take the online multiple choice questions associated with this article (see page 846)
Correspondence to: Dr Tiny Jaarsma, Department of Cardiology, Thoraxcenter, University Hospital Groningen, PO Box 30.001 9700 RB Groningen, The Netherlands; t.jaarsma@ thorax.umcg.nl n most developed countries worldwide, the number of patients with chronic heart failure is growing, with $1-3 \%$ of the adult population suffering from this syndrome, rising to about $10 \%$ in the very elderly. Because the incidence of heart failure increases with age, its prevalence will greatly increase as our population ages. In the near future a large part of the western population will suffer from heart failure and society will be faced with the financial consequences. Heart failure is consuming about $1.5-2.5 \%$ of the health care budget, with approximately $70 \%$ of this being spent on hospitalisation. ${ }^{1}$ On average one in five patients is readmitted within 12 months, making heart failure one of the most common causes of hospitalisation in people over 65 years of age. In a recent international study comparing three states in the USA and three European countries, it was found that among a number of diagnoses, including diabetes, stroke, or hip fracture, the diagnoses of congestive heart failure and chronic obstructive pulmonary disease have the highest hospital readmission rates. ${ }^{2}$

\section{QUALITY OF LIFE}

Not only are readmission rates of heart failure patients higher compared to chronic medical conditions, but also the quality of life of these patients seems to be highly affected. Compared with the healthy general population, heart failure patients show a global reduction in quality of life in several domains (physical functioning, role functioning, and social functioning), reflecting the severe impact of congestive heart failure on daily life, even though some patients are in an ambulatory setting. ${ }^{3}$

Heart failure reduces quality of life more than any other chronic medical condition (for example, diabetes, arthritis, or chronic lung disease) and patients with multiple conditions have greater decrements in functioning and wellbeing than those with only one condition. ${ }^{3}$ This decrease in quality of life is not directly related to functional capacity or systolic function. ${ }^{4}$

Current treatment in heart failure focuses on symptomatic improvement, but also on preventing the transition of asymptomatic cardiac dysfunction to symptomatic heart failure, modulating the progression of heart failure, and reducing mortality. ${ }^{5}$

Despite some recent evidence of improved prognosis after a first hospitalisation for heart failure, pharmacological treatment does not impressively improve the high morbidity and mortality rates associated with chronic heart failure. Mortality rates after a first hospitalisation for heart failure are $11-20 \%$, and one year mortality is also high $(30-45 \%){ }^{1}$

In summary, heart failure is a very common, disabling, and costly disease with a very grim prognosis and poor quality of life. However, a great deal still can be done to improve this situation. In this paper reasons for deterioration of patients and subsequent readmissions will be considered, showing that there is still a lot to gain in terms of decreasing readmissions, morbidity, and mortality, and improving the quality of life of a heart failure patient. This paper also illustrates that the management of heart failure is a complex issue and patient problems are diverse, necessitating a team approach involving different disciplines with differing expertise and skills (fig 1).

WHY IS AN INTER-PROFESSIONAL TEAM APPROACH NEEDED IN HEART FAILURE? Both from a patient's perspective and from the point of view of health care providers, there are a number of reasons why several professionals contribute to optimal and efficient management of heart failure.

\section{Health care providers}

Complexity of diagnosis of heart failure

The diagnosis of heart failure relies on clinical judgement based on a medical history, physical examination, and appropriate investigations. The clinical definition of heart failure is a constellation of symptoms and signs that consist of breathlessness, fatigue, and fluid retention resulting from cardiac dysfunction. ${ }^{5}$ This definition already gives some indication as to how 


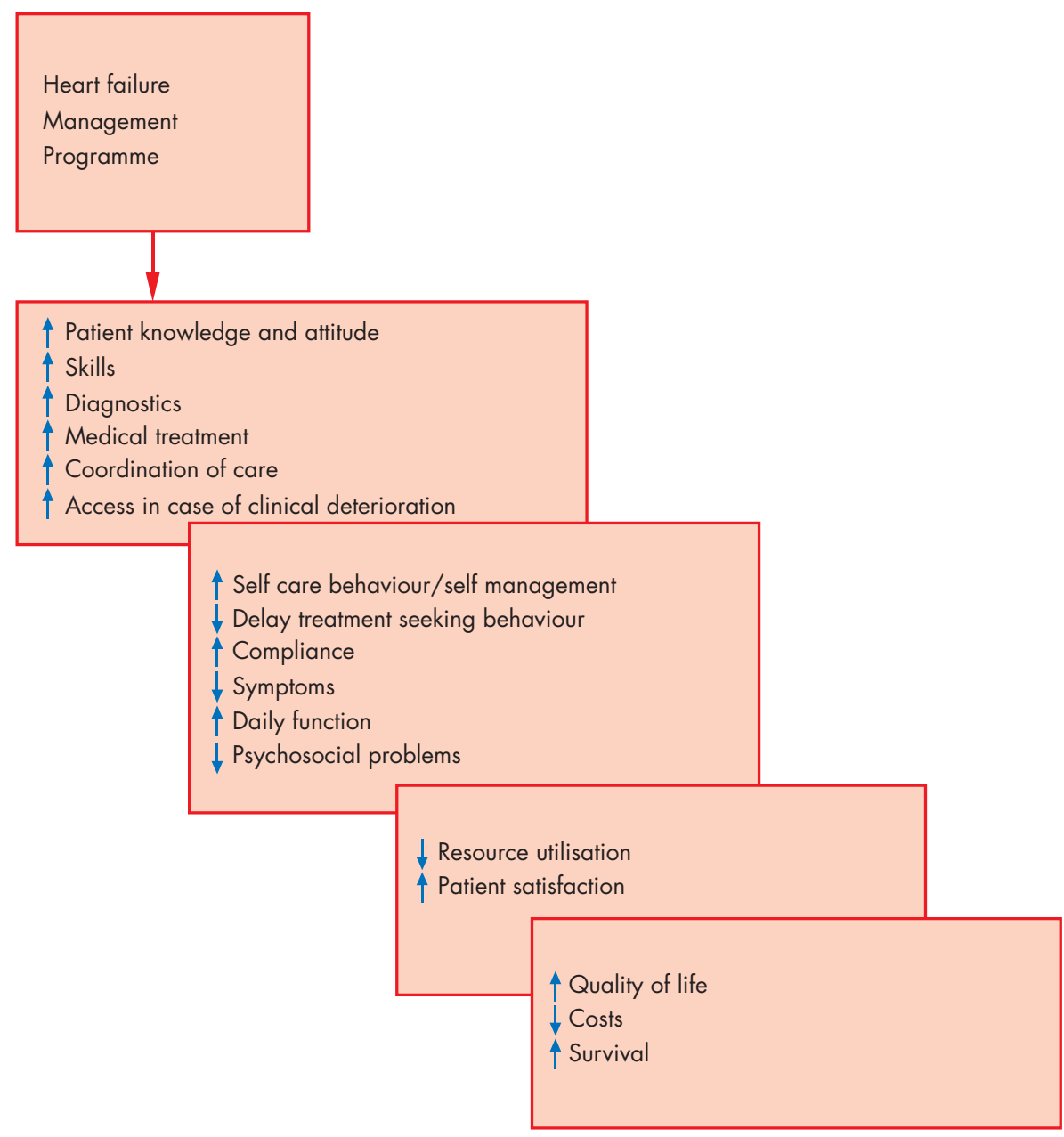

Figure 1 Potential outcomes of heart failure management programmes.

complex the diagnosis of heart failure might be, since currently there is no recognised cut-off value of cardiac or ventricular dysfunction or change in flow, pressure, dimension, or volume that can be used reliably to identify patients with heart failure. The European Society of Cardiology guidelines state: "a patient to be diagnosed with heart failure should have the following features: symptoms of heart failure, typically breathlessness or fatigue, either at rest or during exertion, or ankle swelling and objective evidence of cardiac dysfunction at rest. A clinical response to treatment directed at heart failure alone is not sufficient for diagnosis, although the patient should generally demonstrate some improvement in symptoms and/or signs in response to those treatments where a relatively fast symptomatic improvement could be anticipated e.g. diuretic or nitrate administration." ${ }^{\prime 5}$ Co-morbidities, age, and medical treatment may obscure a diagnosis of heart failure.

General practitioners (GPs), cardiologists, internists, and geriatricians need to cooperate to establish the diagnosis and underlying aetiology of heart failure.

\section{Complexity of heart failure treatment}

Alongside the complexity of diagnosing heart failure, treatment of heart failure has also become increasingly complex. Treatment includes medications that prolong life, alleviate symptoms, and reduce admissions to hospital. ${ }^{5}$ Not only should the right medication be prescribed, but adequate doses should be titrated. In uptitrating drugs in the individual patient, dosages that are advised in guidelines should be aimed for instead of titration based on symptomatic improvement alone. Uptitrating agents on top of other medication sometimes can be a time consuming and precise job, involving several clinical decisions. Other complex decisions in treatment can be related to surgery or device therapy: implantable cardioverter defibrillators (ICDs), biventricular (multi-site) pacing, heart transplantation, or ventricular assist devices. Several professional experts are needed to decide on and administer heart failure treatment.

\section{Co-morbidity}

In most patients, a range of concomitant disorders accompanies heart failure that both contribute to the cause of the disease (for example, hypertension, ischaemia, diabetes mellitus) and have a key role in its progression and response to treatment. ${ }^{6}$ In addition, co-morbid disorders often implicate a medical regimen that includes medication for several disorders and several non-pharmacological treatment aspects (for example, diet and exercise). Co-morbid disorders also enhance the possibility for polypharmacy, which may lead to adverse drug interactions (for example, adding spironolactone to a regimen that includes potassium supplements). Several drugs should be avoided or used with caution when co-prescribed with any form of heart failure treatment (table 1).

Cardiologist, pharmacists, internists, GPs, heart failure nurses, and dieticians can have a role in choosing the appropriate regimen for a patient with several diseases. 
Table 1 Drugs to be avoided in heart failure patients

1. Non-steroidal anti-inflammatory drugs (NSAIDS)

2. Class I antiarrhythmic drugs

3. Calcium antagonists (verapamil, diltiazem, first generation dihydropyridine derivatives)

4. Tricyclic antidepressants

7. Illicit drugs (for example, cocaine, amphetamines)

\section{Patients with heart failure \\ Elderly}

Although the therapeutic approach to heart failure in the elderly should be principally identical to that in younger heart failure patients, treatment should be applied more cautiously. Sometimes reduced dosages are necessary due to, for example, altered pharmacokinetic and pharmacodynamic properties of cardiovascular drugs and renal dysfunction. Changing lifestyle can be more complicated in elderly patients, and introducing a complex and changing medication regimen can be more difficult. ${ }^{7}$ Changes in nutritional habits in elderly patients can lead to reduced calorie/protein intake, possibly resulting in additional complications. Physicians, nurses, and other health care professionals need to combine their efforts and adapt their approach to older, often vulnerable, heart failure patients.

\section{Coping with complex lifestyle changes}

Most patients with heart failure get a complex therapeutic regimen that consists of medication, diet and fluid restriction, daily weighing, and recommendations on activity and rest. The individual patient often has to integrate these lifestyle changes in an already existing medial regimen related to other co-morbid diseases.

Non-compliance with medication and other lifestyle recommendations in heart failure patients is recognised as a major potential problem. Non-compliance can have important consequences for individual heart failure patients such as worsening symptoms, sometimes leading to readmission. ${ }^{7}$ Education and counselling by a multidisciplinary team, with specific team members addressing specific items, has an important role in improving compliance.

\section{Possible impaired cognitive function and/or depression}

Patients with heart failure can suffer from cognitive dysfunction and depression, which will influence educational strategies and compliance with the medical regimen. Forgetfulness or lack of interest can have a major contribution to compliance with treatment or keeping appointments. These complex problems need a patient centred and broad

\section{Need for inter-professional team approach}

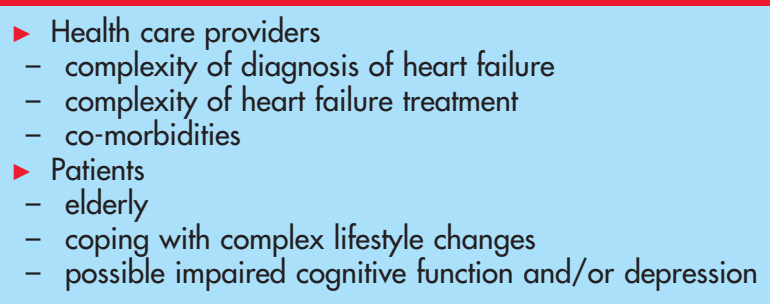

approach, requiring input from physicians, nurses, and other health care providers.

Ignoring the complexity of heart failure for both health care providers and patients can have consequences for patient outcomes. ${ }^{7}$ Table 2 shows factors related to hospital readmission in heart failure patients. It is generally recognised that a large proportion of heart failure readmissions are preventable. Several studies have concluded that readmission could have been avoided in $40-59 \%$ of patients if there had been better assessments, if treatment would have been optimised, if rehabilitation had been more adequate, if discharge had been more carefully planned, if potential non-compliance issues and diet had been identified, and if patients had been instructed to seek medical assistance when symptoms occur. ${ }^{18}$

Summarising, health care practitioners who treat heart failure patients often face the challenge of managing multiple conditions requiring multiple medications and lifestyle changes in an older, sometimes cognitively and psychologically affected patient group. An inter-professional team approach is needed to optimally diagnose, carefully review and prescribe treatment, educate and counsel patients and families in regard to medication use and lifestyle changes, and provide post-discharge follow up.

\section{WHAT IS AN OPTIMAL MODEL TO DELIVER INTER- PROFESSIONAL CARE?}

To date there have been several studies to evaluate the effect of heart failure management programmes designed to improve outcomes in patients with heart failure. These programmes all differ in content and intensity. Although there are a large number of within-model variations, the majority of programmes can be classified as either "heart failure outpatient clinic" or "home based management programme". In addition, combinations of these models or new approaches, like telemonitoring, are used in delivering heart failure care.

Table 2 Reasons for deterioration of heart failure often leading to readmission

Cardiac

- Atrial fibrillation

- Other supraventricular or ventricular arrhythmias

- Myocardial ischaemia

- Appearance of worsening of mitral or tricuspid regurgitation

- Excessive preload reduction (diuretics + ACE inhibitors)

Non-cardiac

$>$ Infection

- Pulmonary embolism

- Co-prescribed drugs

- Alcohol abuse

- Renal dysfunction (excessive use of diuretics)

- Thyroid dysfunction (for example, amiodarone)

- Anaemia (hidden bleeding)

- Inadequate or inappropriate medical treatment

- Discharge in unstable condition

- Adverse effects of prescribed treatment.

- Inadequate knowledge of chronic heart failure and prescribed treatment

- Non-compliance with prescribed treatment

- Inadequate follow up

- Problems with caregivers or extended care facilities

$\checkmark$ Poor social support

$\mathrm{ACE}$, angiotensin converting enzyme. 


\section{Heart failure outpatient clinic}

In a "heart failure clinic" model, care is delivered in an outpatient setting by health care providers with heart failure expertise to patients who attend the clinic. In some clinics the programme starts while the patient is still in the hospital, but the primary site of care delivery is the outpatient clinic. Nurses and cardiologists can have several roles in the heart failure clinic and have a responsibility to bridge the gap between the inpatient and outpatient care. Nurses can play an important role in coordinating or facilitating care. In other clinics nurses have a more independent role in managing and directing care, with primary responsibility for the day-to-day care of patients.

For example, in a heart failure clinic studied by Strömberg and colleagues, the programme consisted of education aimed at assisting patients to improve their self care regimen, nonpharmacological treatment, protocol led changes in medication, psychosocial support, and availability of nurse contact in case of problems. The first heart failure clinic visit was scheduled 2-3 weeks after discharge. ${ }^{9}$ When patients were stable and well informed they were referred back to their GP in primary care. Some other heart failure clinics will keep the patients on regular follow up, without referring them back to their GP.

Reflecting on the advantages and disadvantages of a heart failure clinic, one might say that a heart failure clinic that is situated in an outpatient setting gives the opportunity to have access to medical equipment, specialist consultation, laboratory facilities, and patients' medical charts. At the same time it is known that an outpatient heart failure clinic is often not feasible for elderly and the less mobile patients with moderate to severe, chronic heart failure.

\section{Home based management programmes}

In a home based model, care is delivered primarily in patients' homes and sometimes includes home health facilities, instead of delivering the care in a clinic or outpatient setting. The health care provider comes in person to the home of the patient, calls the patient on the telephone, or both.

For example, in a study in a tertiary referral hospital in Glasgow, patients received multiple home visits and the nurses initiated and titrated pharmacotherapy according to pre-established guidelines. Home based specialist nurses provided comprehensive education and counselling, during an early home visit, and frequent visits and calls. The home based specialist nurse was accessible in case of problems and facilitated communication among health care providers. ${ }^{10}$

Other models for home based care may be less intensive but still include patient education, counselling, and assessment of the need for medication adjustments during a home visit. ${ }^{11}$

The advantage of home based models is that the health care provider has the opportunity to reach vulnerable and frail patients who might not be able to come to a heart failure clinic. Additionally, care is delivered in the non-threatening environment of the patient's home, allowing realistic assessment of the social situation and possible barriers to noncompliance. At the same time it should be realised that home visits might be very time consuming and inefficient in some areas. Furthermore, access to specialist consultation, patient medical records, or medical equipment might be limited in home based models.
Combination of models and integrated care programmes (primary and secondary care)

There are several options to combine components from a heart failure clinic and a home based programme. Some facilities might have the option to deliver both models.

Other models try to integrate primary and secondary care. For example, the Auckland heart failure management study used an integrated management approach involving primary and secondary care in patients with heart failure. Patients received early clinical review, three group education sessions, patient diary, and regular follow up alternating between the GP and the heart failure clinic. ${ }^{12}$

\section{Home telemonitoring}

Telemonitoring can be defined as home monitoring of patients using special telecare devices in conjunction with a telecommunication system. ${ }^{13}$ With technological equipment in the patients' homes, data can be collected on weight, blood pressure, ECG, respiratory rate, transcutaneous $\mathrm{O}_{2}$ saturations, and body temperature.

Telemonitoring can either be used alone or as part of a multidisciplinary approach. Initial studies have suggested that telemonitoring may reduce hospitalisations and readmission rates in patients with heart failure, although more evidence of efficacy is required. ${ }^{13}$

To date, there are no data available that compare the relative cost effectiveness of a clinic versus home based model of care, or address different strategies. Similarly, there are no studies describing patient preferences in this regard. At the same time it is important to note that such programmes are probably most effective when both medical treatment, according to official guidelines, and discharge planning is applied in an optimal manner.

\section{WHO SHOULD BE INVOLVED?}

Heart failure teams can comprise several members depending on the goals of care, the population served, and available resources (finances, personnel, and expertise).

Professionals who can have a role in the comprehensive treatment and care of heart failure patients are: GP, cardiologist, heart failure nurse, home care, internist, dietician, pharmacist, social worker, psychologist, physical therapist, and geriatrician.

Depending on the local health care system, it is important to determine which health care provider is the most appropriate to participate in various components (table 3 ).

\begin{tabular}{l} 
Table 3 Components of multidisciplinary care \\
\hline Appropriate diagnosis \\
- assess severity of symptoms \\
- determine aetiology \\
Optimal medical management \\
Intense education and counselling \\
Discharge planning \\
Vigilant follow up \\
Attention to behavioural strategies \\
Address barriers to compliance \\
Early attention to signs and symptoms (for example, daily weighing, \\
telemonitoring) \\
Flexible diuretic regimen \\
Increased access to health care providers \\
Exercise programme
\end{tabular}


Table 4 List of subjects to discuss with a heart failure patient and his or her family

\section{General advice}

- Explanation of heart failure and why symptoms occur

- Causes of heart failure

- How to recognise symptoms

- What to do if symptoms occur

- Self weighing

- Rationale of treatments

- Importance of adhering to pharmacological and non-pharmacological prescriptions

- Refrain from smoking

- Prognosis

- Advanced directives

Drug counselling

- Nature of each drug, dosing and time of administration

- Side effects and adverse affects

- Signs of intoxication

- What to do in case of skipped doses

- Compliance strategies

Activity, exercise, rest

-Work, leisure activities and travel

- Rest

- Energy conserving suggestions

- Daily physical activity

- Sexual activity

- Rehabilitation: exercise programme

- Compliance strategies

Vaccinations

Symptom assessment and management

- Expected symptoms versus symptoms of worsening heart failure

- Self monitoring of daily weights

- Actions in case of increased symptoms

- Dealing with psychological symptoms

- Self management (for example, diuretics)

Dietary and social habits

- Control sodium intake when necessary

- Avoid excessive fluids in severe heart failure

- Avoid excessive alcohol intake

- Smoking cessation

Compliance strategies

Patients should be diagnosed and treated according to the available guidelines and should be adequately educated (table 4).

The core of the team in a heart failure management programme most often comprises a cardiologist and a heart failure nurse. Together they are responsible for the proper diagnosis, treatment, and care of heart failure patients. The heart failure nurse has a major role in the education and counselling of patients and family, and helping patients when they present signs or symptoms of deterioration. In addition, heart failure nurses can help the patient to learn to live with the consequences of heart failure, which means: comply with a regimen concerning medication, diet and exercise, monitor symptoms, seek assistance when symptoms occur, and take relevant actions in case of exacerbation-for example, to alert a health care provider or adjust their diuretic dose accordingly. There has been a development in the role of the heart failure nurse, leading to more independent function in regard to protocol driven drug titration, referrals, and post-discharge follow up review of patients. However, in most health care systems, ultimately the cardiologist has the final responsibility for the medical management, completeness of investigation, and planning and initiation of the therapeutic strategy.

GPs (primary care physicians) are involved in the patient's course of heart failure at several stages. This starts with the important role in the prevention of heart failure through the early identification of potential aetiological factors and the aggressive management of identified risk factors. Additionally, the GP has a crucial role in the initial diagnosis, implementation of evidence based treatment, and subsequent follow up arrangement, where all activities are aimed at maximising prognosis. ${ }^{14}$

It is known that patients with heart failure visit their GP often with heart failure as the most common reason for consultation. ${ }^{12}$ Independent of the model of the heart failure programme, a close cooperation between primary care and the hospital team should be established.

Dieticians can help to evaluate dietary intake, formulate tailored advice in regard to specific patient needs (for example, combine a heart failure diet with a diabetes diet), and help patients to improve compliance with a prescribed nutrition recommendation. Another important aspect in dietary counselling is the importance of a healthy body weight. Being either overweight or underweight are serious concerns in patients with heart failure. Weight reduction in obese heart failure patients can be a complex issue needing specialised guidance by a dietician. On the other hand, preventing and treating cardiac cachexia may need more attention.

Two studies evaluating the effects of a multidisciplinary approach to heart failure explicitly reported on the role of the dietician. ${ }^{15}{ }^{16}$ However, the role of the dietician is often poorly described ("provision of dietary instructions"). Other minor publications only focus on the individual role of the dietician in heart failure, showing that education by a dietician improves knowledge and awareness and decreases sodium and fluid intake of heart failure patients.

Physical therapists can advise the patient with heart failure on reconditioning and training, and also give practical advice on energy conservation.

Patients that are seen at the heart failure clinic have often been "de-conditioned" by, for example, prolonged bed rest or repeated hospitalisations. Others have been inactive because of fear of increasing symptoms or fear of exertion. These patients possibly can benefit most from exercise programmes, either in a cardiac rehabilitation centre, in a hospital based exercise programme, or in a home exercise programme. Low intensity home walking exercise programmes for patients with stable, moderate heart failure are safe, well accepted, and effective in improving functional status (submaximal exercise capacity) and global perception of symptoms.

Pharmacists already have a well established role in checking on interactions and possible treatment of adverse events when new drugs are initiated. Additional interventions by pharmacists can include providing advice to physicians in choosing the appropriate drug regimen and dosages, and on improving compliance and the patient's knowledge of heart failure. Studies show that patient education and goal setting by pharmacists can increase patient knowledge, the appropriateness of medications taken by patients, and patient compliance. ${ }^{17}$

Psychologists and/or social workers can help patients to cope with the effects of heart failure in their daily life. 
Psychologists can also help doctors and heart failure nurses specifically to look for and identify symptoms of depression in patients with heart failure at an early stage.

In the complex management of heart failure patients, one can imagine that heart failure clinics choose to include other health care professionals in the team. For example, when working with a pre-heart transplant population, close collaboration with transplant coordinators is quite obvious. Working with a chronic and elderly population, some heart failure clinics consider involving geriatricians. Each heart failure team clinic should establish which health care provider will be the obvious person for addressing the specific patient problem.

\section{IS AN INTER-PROFESSIONAL TEAM APPROACH EFFECTIVE?}

A recent meta-analysis by McAllister and colleagues ${ }^{18}$ reported that multidisciplinary management strategies for patients with heart failure are associated with a $27 \%$ reduction in heart failure hospitalisation rates and a $43 \%$ reduction in the total number of heart failure hospitalisations. Those strategies that incorporate specialised follow up by a multidisciplinary team also reduce all cause mortality by approximately a quarter and all cause hospitalisations by a fifth. In this meta-analysis, 29 studies were included that specifically addressed several models of multidisciplinary management.

The authors discuss the elements that should be included in the multidisciplinary management of heart failure patients: the involvement of specially trained heart failure nurses, patient education, and ready access to clinicians trained in heart failure.

Although a wide variety of multidisciplinary strategies exist to manage patients with heart failure, the most optimal model or intensity cannot be determined. Available evidence does not support the assumption of incremental efficacy with more intensive post-discharge intervention. Almost all studies that were evaluated for effectiveness used a multidisciplinary team approach, with teams varying from a cardiologist and a specialised heart failure nurse to a more extended multidisciplinary team caring for heart failure patients. ${ }^{18}$

An example of an intensive comprehensive discharge programme is described by Rich and colleagues, ${ }^{15}$ who reported that a multidisciplinary intervention had beneficial effects as regards rates of hospital readmission, quality of life, and cost of care within 90 days of discharge among "high risk" chronic heart failure patients. The multidisciplinary team consisted of comprehensive education of the patient and family by a heart failure nurse, provision of dietary instructions by a registered dietician, and appropriate postdischarge care by social services. A geriatric cardiologist reviewed each patient's medications and gave specific recommendations to simplify and consolidate the regimens by minimising both the number of medications and the dosing. However, the final choice of medication was left to the discretion of the patient's primary physician. Home care services were supplemented by individual home visits and telephone contact. ${ }^{15}$

More recently McDonald and colleagues ${ }^{16}$ in Ireland also reported on the effects of a multidisciplinary heart failure clinic. In this study the intrinsic benefit of multidisciplinary care in the setting of protocol driven optimal medical management of heart failure was demonstrated. Team members were the cardiology service, the heart failure nurse, and a specialist dietician who consulted patients during the index admission.

\section{CONCLUSION AND OUTLOOK}

An organised system of specialist heart failure care improves outcomes, including quality of life, the frequency and duration of follow up, and survival in patients with heart failure. A multidisciplinary team approach involving several professionals with their own expertise is important in attaining an optimal effect. Physicians, nurses, and other health care professionals are key to ensuring the delivery of evidence based care.

Considering the aging population, an increasing number of heart failure patients will need specialised treatment, care, and guidance in the complex issues of heart failure and comorbidities. As a consequence, the role of individual health care providers is likely to change in the future-for example, more patients will be referred to a heart failure management programme and more patients in a stable condition will be referred back to their GP or primary care provider. In future, the role of the heart failure nurse probably will be more independent from the physician-for example, in regard to optimisation of medical treatment and independent patient follow up. However, one should keep in mind that appropriate training of the health care professionals involved will always be important.

To deliver cost effective care, future treatment and follow up strategies can be expected to become more tailored to the individual patient. Criteria will be developed that will give direction as to what care is needed and which interventions are most effective for a certain group of patients (for example, selecting high and low risk patients). Trials that compare the effects of different models are underway. ${ }^{19}$

Recently the use of B type natriuretic peptide (BNP) has been recognised as being increasingly important in the diagnosis of heart failure and helpful in guiding treatment and monitoring the course of heart failure, particularly in alerting clinicians to decompensation. ${ }^{20}$ Taking this concept further, it can be hypothesised that BNP measurement may also prove a useful addition to clinical assessment in situations where risk stratification is required-for example, to determine which patients may need additional follow up. BNP concentrations might help to determine which patients might be candidates for early follow up and perhaps home nursing visits.

Markers of clinical (in)stability, psychosocial risk factors, and issues related to patient mobility might be important indicators to determine which inter-professional service might be most effective for which patient. In the meantime, health care providers should realise that a multidisciplinary approach cannot be ignored in the provision of optimal treatment and care for heart failure patients.

\section{REFERENCES}

1 McMurray JJ, Stewart S. The burden of heart failure. Eur Heart J 2003;5(suppl):3-14.

2 Westert GP, Lagoe RJ, Keskimaki I, et al. An international study of hospital readmissions and related utilization in Europe and the USA. Health Policy 2002;61:269-78.

3 Stewart AL, Greenfield S, Hays RD, et al. Functional status and well-being of patients with chronic conditions: results from the medical outcomes study. JAMA 1989;262:907-13.

- Study comparing functional status and wellbeing between nine common chronic medical conditions of 9385 patients in the USA. 
4 Jaarsma T, Halfens R, Huijer-Abu Saad H, et al. Quality of life in older patients with systolic and diastolic heart failure. Eur J Heart Fail 1999;1:151-60.

5 Remme WJ, Swedberg K. Guidelines for the diagnosis and treatment of chronic heart failure. Eur Heart J 2001 ; 22:1527-60.

6 Krum H Gilbert RE. Demographics and concomitant disorders in heart failure. Lancet 2003;362:147-58.

7 Wal van der MHL, Jaarsma T, Veldhuisen van DJ. Non-compliance in patients with heart failure; how can we manage it? Eur $J$ Heart Fail 2005;7:81-6.

838 Literature review on the degree and the consequences of noncompliance in heart failure, and possible interventions.

8 Mosterd A, Hoes AW. Reducing hospitalizations for heart failure. Eur Heart J 2002;23:842-5.

9 Stromberg A, Martensson J, Fridlund B, et al. Nurse-led heart failure clinics improve survival and self care behavior in patients with heart failure. Results from a prospective, randomized trial. Eur Heart J 2003;24:1014-23.

- First study to find significant effects on mortality of a heart failure clinic.

10 Blue L, Strong E, Murdoch DR, et al. Improving long-term outcome with specialist nurse intervention in heart failure: a randomized trial. $B M J$ 2002;323:1112-15.

- First randomised study to examine a programme of care where specialised nurses, in addition to providing education and counselling, initiated and titrated pharmacotherapy during home visits.

11 Stewart S, Horowitz JD. Home-based intervention in congestive heart failure: long-term implications on readmission and survival. Circulation 2002;105:2861-6.

- This study reports long term follow up of patients after a multidisciplinary home based intervention with a post-discharge home visit at 7-14 days.

12 Doughty RN, Wright SP, Walsh HJ, et al. Randomised, controlled trial of integrated heart failure management: the Auckland heart failure management study. Eur Heart J 2002;23:139-46.

- In this study, integration between primary and secondary care was attempted. Authors also describe frequent visits to the GP.

13 Louis AA, Turner T, Gretton M, et al. A systematic review of telemonitoring for the management of heart failure. Eur J Heart Fail 2003;5:583-90.
14 Hobbs FD. Primary care physicians: champions of or an impediment to optimal care of the patient with heart failure? Eur J Heart Fail 1999;1:11-5.

15 Rich MW, Beckham V, Wittenberg C, et al. A multidisciplinary intervention to prevent readmission of elderly patients with congestive heart failure. N Engl J Med 1995;333:1190-5.

- First properly powered randomised study of a multidisciplinary intervention in heart failure.

16 McDonald K, Ledwidge M, Cahill J, et al. Elimination of early rehospitalization in a randomized, controlled trial of multidisciplinary care in a high-risk, elderly heart failure population: the potential contributions of specialist care, clinical stability and optimal ACE-inhibitor dose at discharge. Eur J Heart Fail 2001;3:209-15.

- Irish study reporting a very high success of a multidisciplinary intervention.

17 Gattis WA, Hasselblad V, Whellan DJ, et al. Reduction in heart failure events by the addition of a clinical pharmacist to the heart failure management team: results of the pharmacist in heart failure assessment recommendation and monitoring (PHARM) study. Arch Intern Med 1999;59:1939-45

- Randomised study describing the addition of a clinical pharmacist to the heart failure management team.

18 McAlister FA, Stewart S, Ferrua S, et al. Multidisciplinary strategies for the management of heart failure patients at high risk for admission. A systematic review of randomized trials. J Am Coll Cardiol 2004;44:810-9.

- Meta-analysis of 29 studies that specifically addressed multidisciplinary strategies for the management of heart failure patients.

19 Jaarsma T, Van Der Wal MH, Hogenhuis J, et al. Design and methodology of the $\mathrm{COACH}$ study: a multicenter randomised coordinating study evaluating outcomes of advising and counselling in heart failure. Eur $J$ Heart Fail 2004;6:227-33.

20 Cowie MR, Jourdain P, Maisel A, et al. Clinical applications of B-type natriuretic peptide (BNP) testing. Eur Heart J 2003;24:1710-8.

- Summarises the current evidence on BNP and provides guidance for practising clinicians. 